\title{
Solitary primary intracranial leptomeningeal glioblastoma invading the normal cortex: Case report
}

\author{
TAKAMICHI KATSUHARA ${ }^{1}$, NOBUHIRO MORO ${ }^{1}$, TAKASHI OHTA $^{1}$, TAKU HOMMA $^{2}$ and ATSUO YOSHINO ${ }^{1}$ \\ Departments of ${ }^{1}$ Neurological Surgery and ${ }^{2}$ Pathology, Nihon University School of Medicine, Tokyo 173-8610, Japan
}

Received October 18, 2017; Accepted January 22, 2018

DOI: $10.3892 / \mathrm{mco} .2018 .1561$

\begin{abstract}
Solitary primary intracranial leptomeningeal glioma (PLG) is a rare entity of glioma. PLG arises from the heterotopic glial tissue in the subarachnoid space and usually grows there without parenchymal invasion. The present study reported a case of solitary PLG, pathologically diagnosed as glioblastoma, that invaded the temporal cortex and finally disseminated to the spinal cord. A 55-year-old woman had headaches and visited Nihon University, Itabashi Hospital. Head magnetic resonance imaging showed a solid mass mainly located in the right middle fossa extending to the frontal base with strong enhancement effect after contrast medium injection. A conventional angiogram showed a tumor arising from the middle meningeal artery. Fronto-temporal craniotomy was performed to remove the tumor. During reflection of the dura matter, there were numerous small vessels connecting the dura matter and the cortical surface. The tumor was located in the Sylvian fissure and extended around the middle cerebral artery. The border between the tumor and the normal temporal lobe was unclear. Temporal lobectomy was done, but the tumor was left around the perforators of the middle cerebral artery. Hematoxylin and eosin staining showed typical glioblastoma with high cellularity, mitosis, pseudopallisading and vascular proliferation. The tumor cells were immunohistochemically negative for isocitrate dehydrogenase (IDH)1-R132H indicating glioblastoma, IDH-wild type. The patient received chemotherapy and radiation therapy, and was discharged from the hospital. Six months later, local regrowth and spinal
\end{abstract}

Correspondence to: Dr Nobuhiro Moro, Department of Neurological Surgery, Nihon University School of Medicine, 30-1 Oyaguchikamimachi, Itabashi, Tokyo 173-8610, Japan

E-mail: moro.nobuhiro@nihon-u.ac.jp

Abbreviations: CT, computerized tomography; MRI, magnetic resonance imaging; PLG, primary intracranial leptomeningeal glioma; IDH, isocitrate dehydrogenase

Key words: solitary primary intracranial leptomeningeal glioma, glioblastoma, parenchymal invasion, heterotopic glial cluster, immunohistochemically negative for isocitrate dehydrogenase 1 dissemination were found. Despite additional chemotherapy and radiation therapy, the tumor became uncontrollable and the patient succumbed. Only 15 cases of solitary PLGs have been reported previously. The IDH status of these tumors have not been investigated in most cases; however, pathological grading varies from lower to higher grade glioma. Together with the pathological difference of astrocytic or oligodendrocytic tumors, solitary PLGs may develop due to various gene alterations similar to intra-axial gliomas.

\section{Introduction}

Primary intracranial leptomeningeal gliomas (PLG), is a rare entity that arises from the heterotopic glial tissue in the subarachnoid space as first described by Wolbach (1). PLGs have been divided into two different forms, solitary and diffuse. Diffuse PLG is often difficult to exclude the exophytic growth from the underlying brain or seeding from a distant primary lesion in the central nervous system $(2,3)$. On the other hand, solitary PLG is extremely rare that only 16 cases including the present case have been reported in the literature, on basis of currently available data (2-14). We herein report a case of intracranial focal solitary PLG, which mimicked an extra-axial tumor, to better characterize this rare tumor.

\section{Case report}

History and examination. A 55-year-old woman who had suffered from headache and nausea for a few weeks admitted to our hospital. She had no obvious abnormality on neurological examinations. Her past medical history was not appreciable except for thymoma removal surgery in her forties, and her family history was unremarkable. Informed consent was obtained from the patient and her family. Magnetic resonance imaging (MRI) demonstrated a brain tumor occupying the tip of middle fossa to the frontal base with a maximal diameter of $25 \mathrm{~mm}$. The greater part of the lesion showed low signal intensity on T1 weighted images and iso signal intensity on T2 weighted images. Middle cerebral artery was involved by the tumor. A massive perifocal edema was found in the adjacent brain. The lesion was strongly and heterogeneously enhanced after contrast medium injection (Fig. 1). Internal carotid artery angiography did not show any abnormality. However, external carotid artery angiography showed a tumor stain from the middle meningeal artery in where the mass was located on 


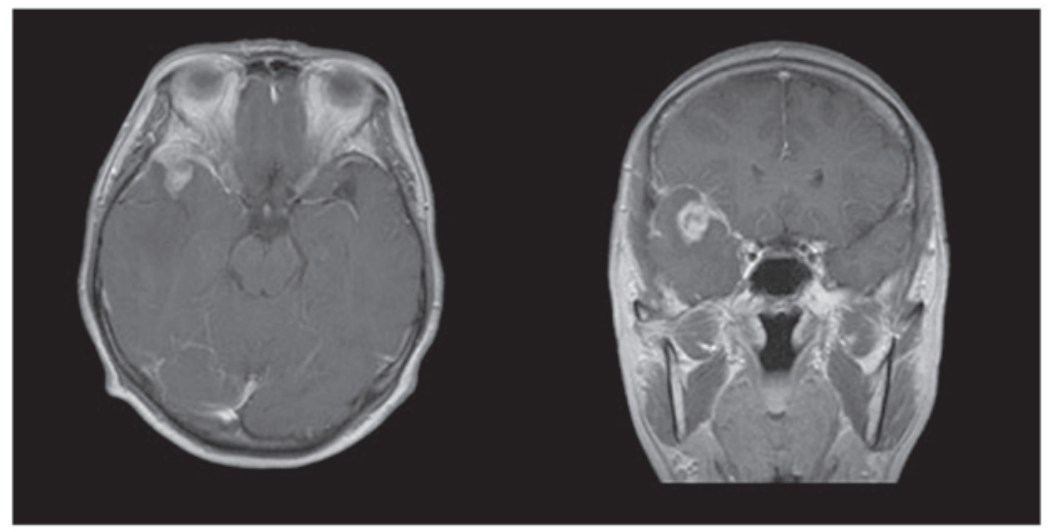

Figure 1. Pre-surgical MRI. Gadolinium-enhanced axial and coronal T1-weighted images showing well-circumscribed tumor mass located from the right middle fossa to the frontal skull base, without evidence of intra-axial involvement.

MRI (Fig. 2). Such radiological findings were interpreted as diagnostic hemangiopericytoma or meningioma.

Surgery. A frontotemporal craniotomy was made. Dura matter was adhered to the surface of the temporal cortex and was not easy to separate. This connecting tissue was coagulated and cut one by one to flip the dura matter under microscopy. Numerous small vessels were found within this connective tissue that easily bled. The tumor was found immediately after opening the Sylvian fissure, and was distinguished from the surrounding brain parenchyma in this region. The tumor was soft and easily bled. As opening the Sylvian fissure further, the M1 segment of the middle cerebral artery was found to be involved within the tumor mass. Tumor was removed as much as possible but avoided removing the tumor involving the perforators of the middle cerebral artery. When reaching deeply into the Sylvian fissure, the tumor infiltrated into the temporal lobe and there was no clear border between the tumor and the adjacent normal parenchyma. Therefore, right temporal lobectomy was made at $50 \mathrm{~mm}$ from the temporal tip to remove the entire mass.

Histopathological examination. The specimen exhibited nuclear and cytoplasmic pleomorphism and nuclear atypia. Highly atypical astrocytes with numerous mitotic nuclei were found overall. Necrosis with pseudopalisading and microvascular proliferation were seen in several areas (Fig. 3). The tumor cells showed intense staining for glial fibrillary acidic protein indicating their astrocytic nature and high Ki-67 proliferative index of $\geq 40 \%$. An obvious invasion of the tumor into the temporal lobe was observed. The tumor cells were immunohistochemically negative for isocitrate dehydrogenase (IDH)1-R132H and p53, and positive for epidermal growth factor receptor. Feature of hemangiopericytoma was not seen all over the section with negative silver staining. Therefore, the pathological diagnosis was determined to be a glioblastoma, IDH-wild type, a glioma of World Health Organization grade IV, originating from a heterotopic glial cluster in the vicinity of the right Sylvian cistern.

Post-operative course. Under diagnosis as glioblastoma, the tumor bed was irradiated with $60 \mathrm{~Gy}$ in 30 times of fraction. She also received administration of temozolomide chemotherapy

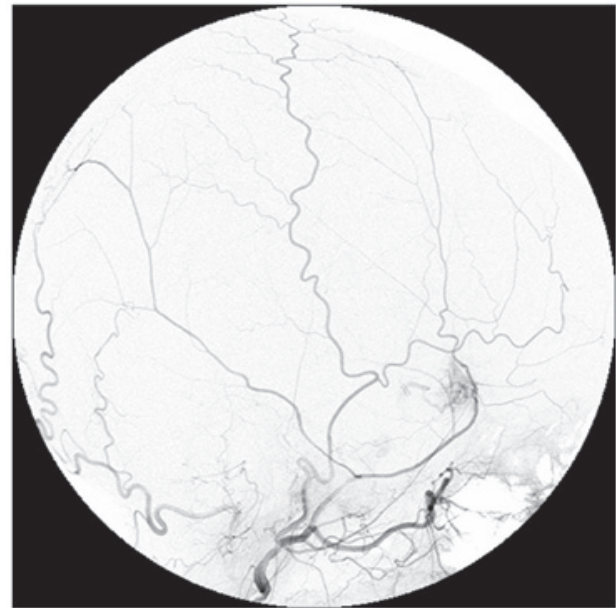

Figure 2. Pre-surgical angiography. Right external carotid arteriograms demonstrating a marked vascular supply via hypertrophic branches of the right middle meningeal artery.

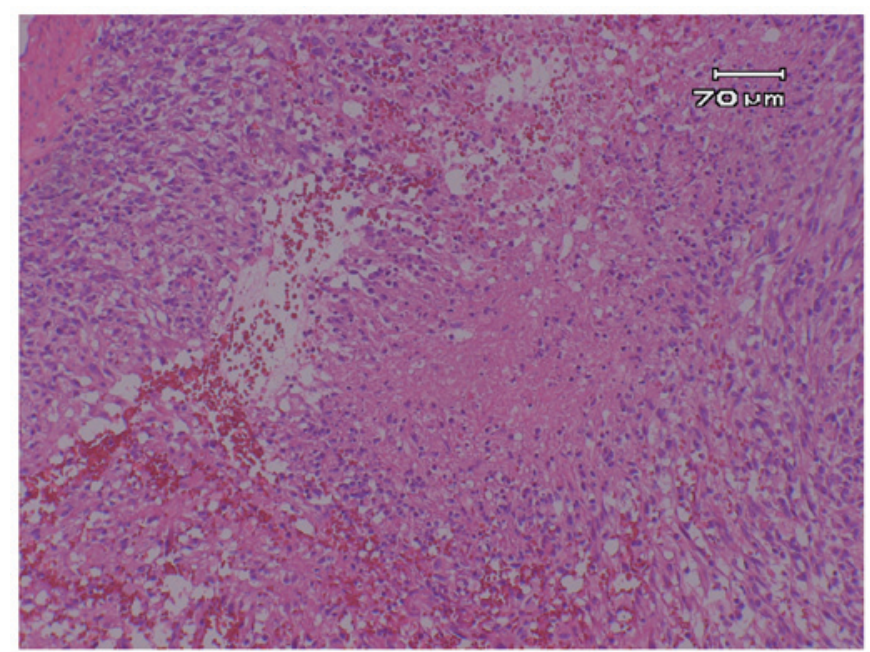

Figure 3. Hematoxylin and eosin staining of the tumor. Intra-tumoral necrosis with pseudopalisading and microvascular proliferation. Scale bar, $70 \mu \mathrm{m}$.

according to the Stupp regimen. The patient was discharged from the hospital without any neurological deficits. 


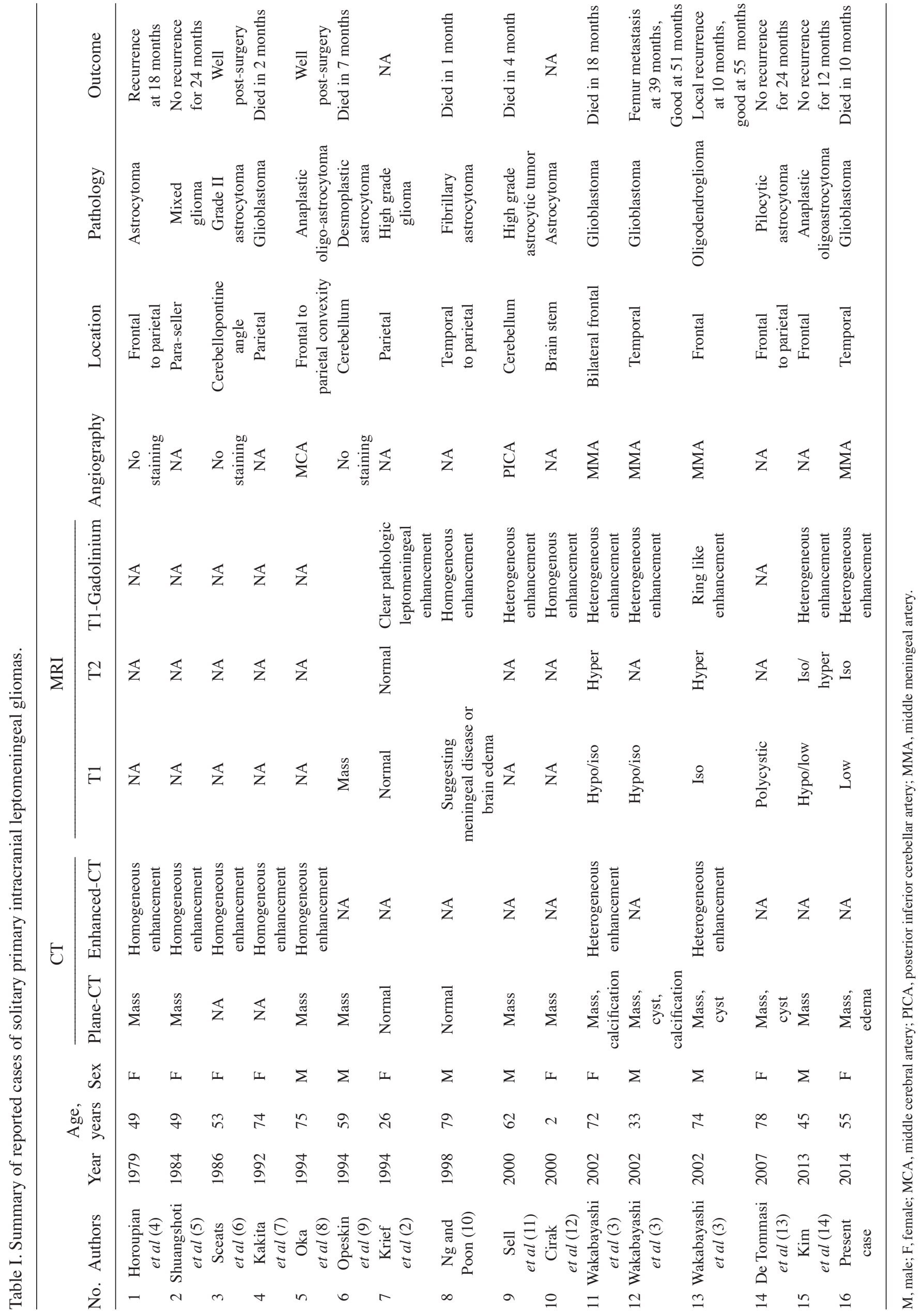


Six months after the initial surgery, the patient complained motor weakness in both lower limbs. Spinal MRI revealed multiple enhanced masses throughout the spinal cord, consistent with dissemination. Brain MRI also indicated a local re-growth of the original tumor in where the tumor was left. A second angiography was performed, but no tumor stain was seen from both internal and external carotid arteries. Additional radiation against the spinal lesions and advanced chemotherapy were done but the tumor became uncontrollable. The patient died 10 months after the initial surgery. Autopsy was not performed.

\section{Discussion}

Solitary PLG is extremely rare and there is no consensus on the diagnostic criteria or treatment. The pathogenesis of solitary PLG remains unclear, and it is speculated that it originates from the leptomeningeal heterotopic neuroglial nests that separated from the bulk of the central nervous system during embryogenesis and subsequently underwent neoplastic transformation in rare instances $(15,16)$. Heterotopic neuroglial nests in the subarachnoid space are seen in $\sim 1 \%$ of normal individuals; the incidence is higher $(25 \%)$ in patients with various congenital malformations of the brain and spinal cord (16). However, it remains unclear, particularly with respect to diffuse PLG, whether such lesions should be considered genuine primitive tumor or as seeding of unknown intra-parenchymal glioma (2). More rarely they were reported as solitary and focal tumor form with or without leptomeningeal spreading, mimicking an extra-axial central nervous system tumor such as meningioma, acoustic neurinoma, or metastasis $(6,11)$. From the basis of currently available data, descriptive information summarizing the previously reported and described cases of solitary and focal form of PLGs are listed in Table I.

Radiological features of intracranial solitary PLGs are not well documented, but frequently observed as extra-axial tumors mimicking meningioma. Computerized tomography (CT) findings were reported in 14 cases. From the CT scan findings, most of the authors indicated an iso density mass only available to identify the tumor. But some tumors included intra-tumoral high density or cystic component, indicating calcification and cyst (3). Enhanced CT was taken in 8 cases and most of the tumors showed homogeneous enhancement. MRI was taken in 10 cases. MRI after gadolinium injection showed that most tumors were enhanced but in several patterns. One case reported by Wakabayashi et al (3) presented a ring-like enhancement effect, which was a characteristic enhancing pattern of an intra-axial glioma. The same authors indicated that careful analysis of MRI patterns suggested glioma rather than meningioma in their cases. Taken together, such CT and MRI patterns seems to depend on the pathological characteristics and malignancy of the tumor.

From the previous description of angiography of 8 cases out of 15 previous cases, which vessel gives blood supply to solitary PLG seems controversial. Three tumors were fed by the branches of the external carotid artery and one from the internal carotid artery (but not from the external carotid artery). One solitary PLG in the posterior fossa was fed by the posterior inferior cerebellar artery. The remaining 3 cases did not show tumor staining from any arteries. In the present case, tumor stain was clearly seen from the middle meningeal artery but not from the internal carotid artery. This vascular supply was consistent with the intra-operative finding that numerous capillary vessels connecting the dura matter and the tumor. The presence of tumor staining again seems to rely on the pathological malignancy of the tumor. Indeed, 3 cases that did not show any tumor stain were all diagnosed pathologically as lower grade glioma. In conclusion, because the pathology of solitary PLGs can vary from astrocytic to oligodendrocytic glioma, both from lower grade to higher grade, it makes it different to define the characteristics by any radiological diagnostic tools.

The PLG was first described by Wolbach in 1907 (1). Cooper and Kernohan in 1951 suggested that meningeal gliomas have no apparent attachment or neoplastic process into the brain or spinal cord in any cases (16). Therefore, it might be emphasized as a tumor that grows in the subarachnoid space without any invasion into the brain parenchyma, although there is no consensus on the diagnostic criteria for such rare neoplastic condition (14). However, it was mentioned that lesions were sometimes linked to the parenchyma by glial bridges extending via the perivascular spaces into the superficical layers of the cortex. Krief et al (2) reported a case of solitary PLG that showed small pathological cortical involvement that was not seen by pre-surgical MRI. Further, some reports indicated a tendency of intra-operative parenchymal invasion $(3,8)$. In the present case, the diagnosis of intracranial solitary PLG was made based on the evidence that the pre-surgical angiogram showing tumor stain from the middle meningeal artery and because the majority of the tumor existed in the subarachnoid space although the tumor obviously invaded the temporal parenchyma confirmed by both intra-operative and pathological finding. In the present case, tumor probably originated from a heterotopic glial cluster in the vicinity of the right Sylvian cistern. The present case shows that solitary PLG might invade the parenchyma, disseminate to the spinal cord, and make multiple lesions depending on the tumor character. The present tumor was IDH-wild type glioblastoma but genomic details are not analyzed in the previous reports. IDH status might vary among solitary PLG cases considering the wide variety of pathological feature.

\section{Competing interests}

The authors declare that they have no competing interests.

\section{References}

1. Wolbach SB: Congenital Rhabdomyoma of the Heart. J Med Res 16: 495-520.7, 1907.

2. Krief O, Monnier L, Cornu P, Foncin JF, Dormont D and Marsault C: MR of isolated leptomeningeal glioma. AJNR Am J Neuroradiol 15: 1782-1784, 1994.

3. Wakabayashi K, Shimura T, Mizutani N, Koide A, Yamagiwa O, Mori F, Nishiyama K, Tanaka R and Takahashi H: Primary intracranial solitary leptomeningeal glioma: A report of 3 cases. Clin Neuropathol 21: 206-213, 2002.

4. Horoupian DS, Lax F and Suzuki K: Extracerebral leptomeningeal astrocytoma mimicking a meningioma. Arch Pathol Lab Med 103: 676-679, 1979.

5. Shuangshoti S, Kasantikul V, Suwanwela N and Suwanwela C: Solitary primary intracranial extracerebral glioma. Case report. J Neurosurg 61: 777-781, 1984.

6. Sceats DJ Jr, Quisling R, Rhoton AL Jr, Ballinger WE and Ryan P: Primary leptomeningeal glioma mimicking an acoustic neuroma: Case report with review of the literature. Neurosurgery 19: 649-654, 1986. 
7. Kakita A, Wakabayashi K, Takahashi H, Ohama E, Ikuta F and Tokiguchi S: Primary leptomeningeal glioma: Ultrastructural and laminin immunohistochemical studies. Acta Neuropathol 83: 538-542, 1992

8. Oka H, Kawano N, Morii S, Suwa T, Irikura K and Saitoh T: Intracranial extracerebral glioma]. Noshuyo Byori 11: 193-200, 1994. Noshuyo Byori 11: 193-200, 1994 (In Japanese).

9. Opeskin K, Anderson RM and Nye DH: Primary meningeal glioma. Pathology 26: 72-74, 1994.

10. Ng HK and Poon WS: Primary leptomeningeal astrocytoma. Case report. J Neurosurg 88: 586-589, 1998.

11. Sell M, Mitrovics T and Sander BC: Primary nodular meningeal glioma mimicking metastatic tumor of the cerebellum with diffuse infra- and supratentorial leptomeningeal spread. Clin Neuropathol 19: 126-130, 2000.

12. Cirak B, Caksen H, Ugras S and Unal O: Primary leptomeningeal astrocytoma in a child. Pediatr Int 42: 389-391, 2000.

13. De Tommasi A, Occhiogrosso G, De Tommasi C, Luzzi S, Cimmino A and Ciappetta P: A polycystic variant of a primary intracranial leptomeningeal astrocytoma: Case report and literature review. World J Surg Oncol 5: 72, 2007.
14. Kim YG, Kim EH, Kim SH and Chang JH: Solitary primary leptomeningeal glioma: Case report. Brain Tumor Res Treat 1: 36-41, 2013.

15. Bailey P and Robitaille Y: Primary diffuse leptomeningeal gliomatosis. Can J Neurol Sci 12: 278-281, 1985.

16. Cooper IS and Kernohan JW: Heterotopic glial nests in the subarachnoid space; histopathologic characteristics, mode of origin and relation to meningeal gliomas. J Neuropathol Exp Neurol 10: 16-29, 1951. 